\title{
Performance Analysis of Flooding and SPIN in Wireless Sensor Networks
}

\author{
Kalpana Sharma ${ }^{1}$, Neha Mittal $^{2}$ and Priyanka Rathi ${ }^{3}$ \\ ${ }^{1}$ Associate Professor, Department of Computer Science and Engineering \\ Sikkim Manipal Institute of Technology \\ Sikkim-737136, India \\ ${ }^{2}$ Department of Computer Science and Engineering \\ Sikkim Manipal Institute of Technology \\ Sikkim-737136, India \\ ${ }^{3}$ Department of Computer Science and Engineering \\ Sikkim Manipal Institute of Technology \\ Sikkim-737136, India \\ ${ }^{1}$ kalpanaiitkgp@yahoo.com, ${ }^{2}$ nehamittal135@gmail.com, \\ 3priyanka.rathi7792@gmail.com
}

\begin{abstract}
Wireless Sensor Networks (WSNs) have many capabilities like wireless communications, sensing and computation. Due to these advanced features they perform critical tasks in many application areas such as environment monitoring, health applications, rescue operations and many more. One of the major challenges in WSN is efficient data transmission and to achieve this many energy efficient routing protocols have been proposed. Flooding is one of the major communication techniques in this aspect. However it suffers from many problems like implosion, overlap and resource blindness [25]. These problems can be addressed using data-centric data transmission approach. One of the routing protocols employing this approach is Sensor Protocols for Information via Negotiation (SPIN) protocol [2] which uses meta-data to eliminate the transmission of redundant data throughout the network. There are four different SPIN protocols namely SPIN-PP for point to point transmission, SPIN-EC which adds energy conservation heuristic [7], SPIN-BC which is used for broadcast transmission media and $S P I N-R L$ which is a reliable version of SPIN-BC. In this paper a comparative study has been done among flooding and the SPIN protocols.
\end{abstract}

Keywords: SPIN, Flooding, Meta-data, Sensor Network, WSN

\section{Introduction}

Many small, lightweight wireless nodes called sensors [11] come together to monitor large physical environments or system to form a wireless sensor network.It is done by measuring the physical parameters such as temperature, pressure, humidity, sound, vibration, pollutants and collectively sending their sensed data to the sink node. Sensor nodes communicate not only with each other but also with a Base Station (BS) using their wireless radio. This allows them to spread their sensor data to 
remote processing, visualization, analysis, and storage systems [4]. The sensor nodes may be simple to monitor a single physical phenomenon or complex, which may combine many different sensing techniques (e.g., acoustic, optical, magnetic) [8].

There has been a huge increase in the use of wireless sensor networks in the recent years due to their ability to monitor and control the physical environment from remote locations [13]. They can greatly improve the accuracy of information obtained through collaboration among sensor nodes and online processing of information. Wireless sensor networks can also improve remote access to sensor data by providing sink nodes that connect them to other networks, such as the Internet, using wide-area wireless links. In wireless sensor networks there are many challenges like energy constraint, design constraint, self management, security, etc., [12]. Apart from sensing ability WSNs also have many advantages like onboard processing, communication and storage capability because of which it finds usage in many applications like environment monitoring, target tracking, healthcare, transportation and many more. Various researches have also been carried out in this area [29].

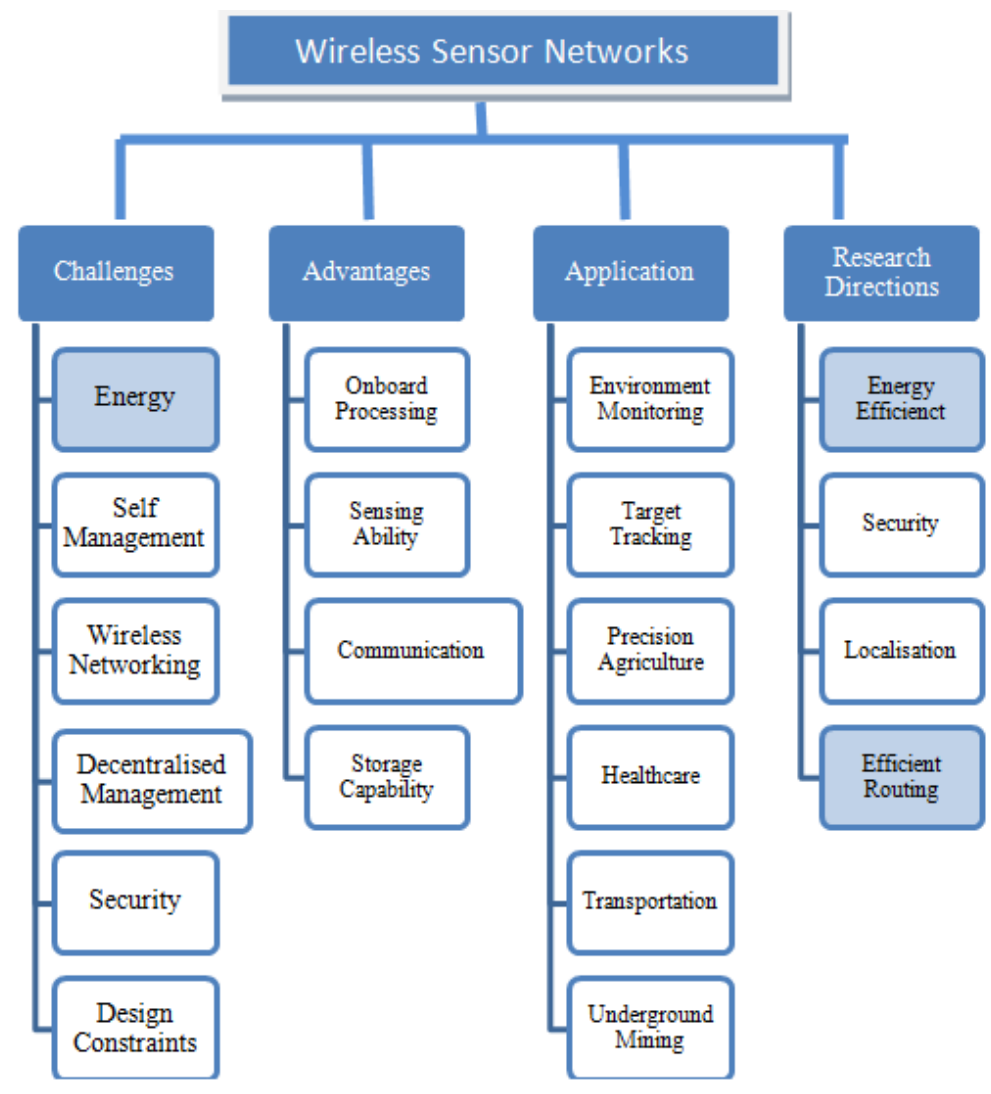

Figure 1. Overview of Wireless Sensor Networks

One of the major issues in WSNs is to develop an energy efficient routing protocol which has a significant impact on the overall lifetime of the network. It suffers from various obstacles arising from limited energy [5], computational power and communication resources available to the sensors in the network. 


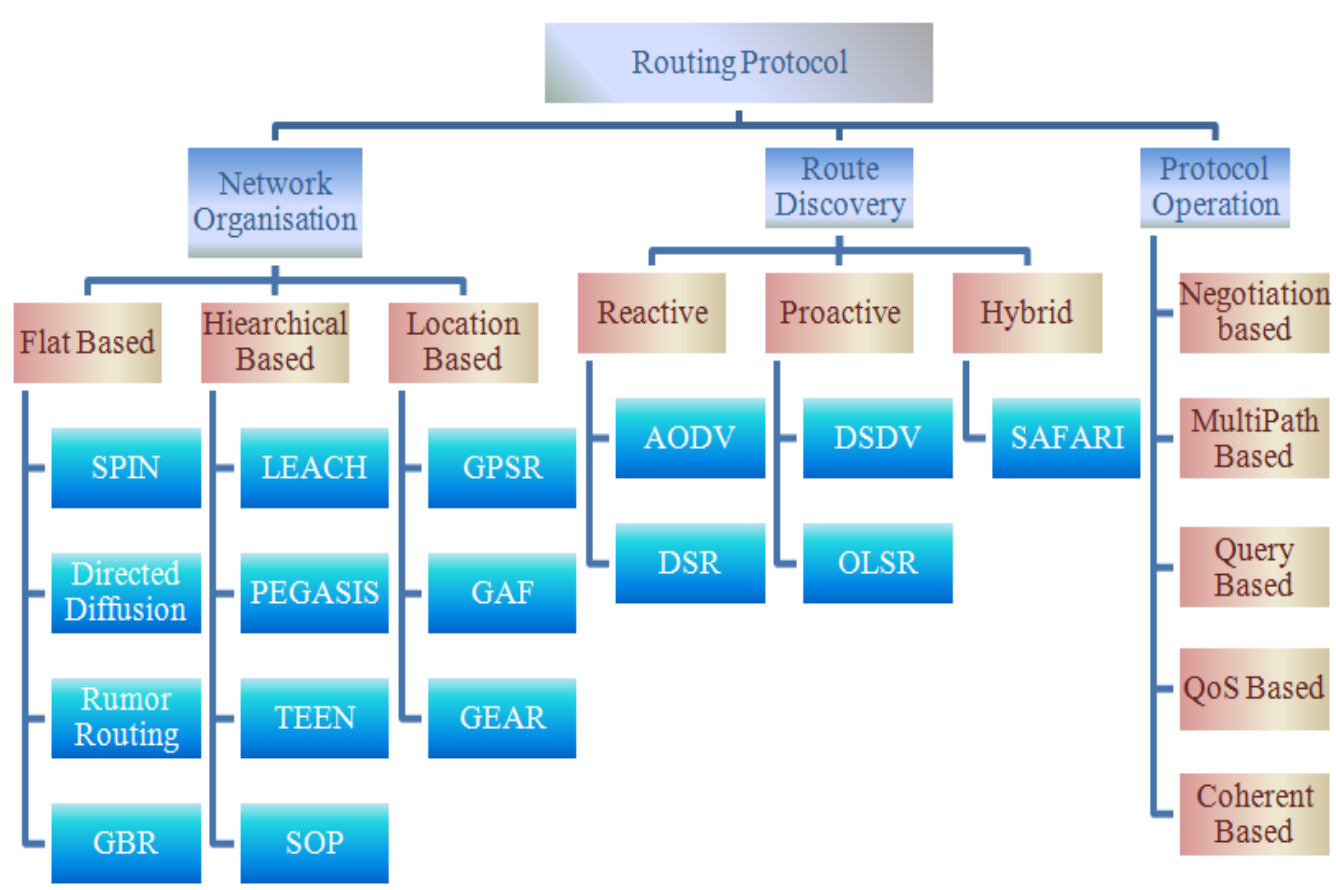

Figure 2. Different Routing Protocols

There are many ways to classify routing protocols. Figure 2 shows three different classifications based on network organisation, route discovery and protocol operation [20]. With respect to network organisation, there are three common classes of routing protocols: Sensor Protocol for Information via Negotiation (SPIN) [1], Directed diffusion, Rumor routing and Gradient Based Routing (GBR) are the examples of flat based routing protocols which assigns equal role to all the nodes. However hierarchical based routing protocols assume different roles for different nodes. This family consists of many protocols some of which are Low Energy Adaptive Clustering Hierarchy (LEACH), Power Efficient Gathering in Sensor Information Systems (PEGASIS), ThresholdSensitive Energy Efficient-Sensor Network (TEEN), and Self Organizing Protocol (SOP). Location based routing protocols rely on the location information from nodes to make routing decisions. Some Location based routing protocols are Greedy Perimeter Stateless Routing (GPSR), Geographic Adaptive Fidelity (GAF), Geographic and Energy Aware Routing (GEAR). Some protocols under reactive routing are Ad Hoc On-Demand Distance Vector (AODV) and Dynamic Source Routing (DSR) while DestinationSequenced Distance Vector (DSDV) and Optimised Link State routing (OLSR) are examples of Proactive Routing. The Hybrid routing protocols exhibit features of both reactive and proactive protocols like Safari. Finally, routing protocols also differ in their operation, for example, negotiation based, multipath based, query based, Quality of Service (QoS) and coherent based protocols [17].

The design of the routing protocol depends on the nature of the application requirements.The routing protocols used earlier were address centred where packets were routed based on unique IP address and the data content remained unchanged during the data delivery process. But this type of addressing scheme is not suitable for WSN, because it is hard to identify the sensor nodes in the network. Since most WSNs are application specific [13] it is relatively advantageous to concentrate on data content rather that address. Data-centric routing is one of them [16]. In data-centric routing scheme, data are retrieved through querying. It is based on certain attribute values like advertisement of data or interest for data which is propagated throughout the network. Moreover, local data are aggregated and it is also possible to add new data at different levels of hop. There are 
two popular approaches in this context- SPIN [10,9] family of negotiation protocol and Directed Diffusion [9]. In this paper we present a comparative study between flooding and the SPIN protocol and its various types. The use of SPIN protocol will result in less amount of packet transmission which will gradually save a significant amount of energy. The rest of the paper is organized as follows. In Section 2, a description of flooding is given. The Spin protocol is described in Section 3. Related work has been discussed in Section 4. Performance Analysis of SPIN and flooding has been done in Section 5, followed by conclusion in Sections 6 .

\section{Flooding}

Flooding is a simple routing algorithm in which every incoming packet is sent through every outgoing link except the one it arrived on. It is an old and simple strategy to spread information into a network or to reach a node at an unknown location by flooding the entire network [23]. A sender node broadcasts packets to its immediate neighbours, which in turn will repeat this process by rebroadcasting the packets to their own neighbours until all nodes have received the packets. Flooding ensures that if there is a path to the destination, it is guaranteed to receive the data. The main advantage of flooding is its simplicity, while its main disadvantage is that it causes heavy traffic. Therefore, measures must be taken to ensure that packets do not travel throughout the network indefinitely. For example, maximum-hop counts are used to limit the number of times a packet is forwarded. It should be large enough so that every intended receiver can be reached, but also small enough to ensure that packets do not travel too long in the network. Sequence numbers in packets can also be used to uniquely identify a packet. In this case whenever a node receives a packet that it has already forwarded, it simply discards the duplicate. However, even with these mechanisms, flooding faces a number of additional challenges [29] as mentioned below.

\section{- Implosion}

In flooding, a node always sends data to its neighbor nodes even if the neighbor has already received the data from another source. It results in the implosion problem depicted in the figure below. Here, node A starts by flooding data to its two neighbors, B and $\mathrm{C}$. These nodes store the data received from A and send a copy of it to their neighbor D. The protocol, thus, wastes resources by sending two copies of the data to D.

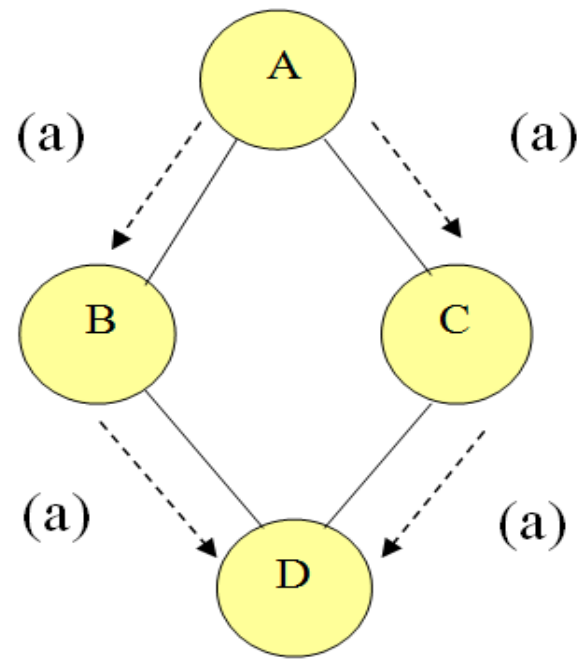

Figure 3. Implosion [25] 


\section{- Overlap}

Sensor nodes often cover overlapping geographic areas due to which nodes gather overlapping data. Figure below illustrates what happens when two nodes (A and B) gather such overlapping data and then flood the data to their common neighbor (C). Similarly the flooding algorithm in this case too, wastes energy and bandwidth by sending two copies of a piece of data to the same node.

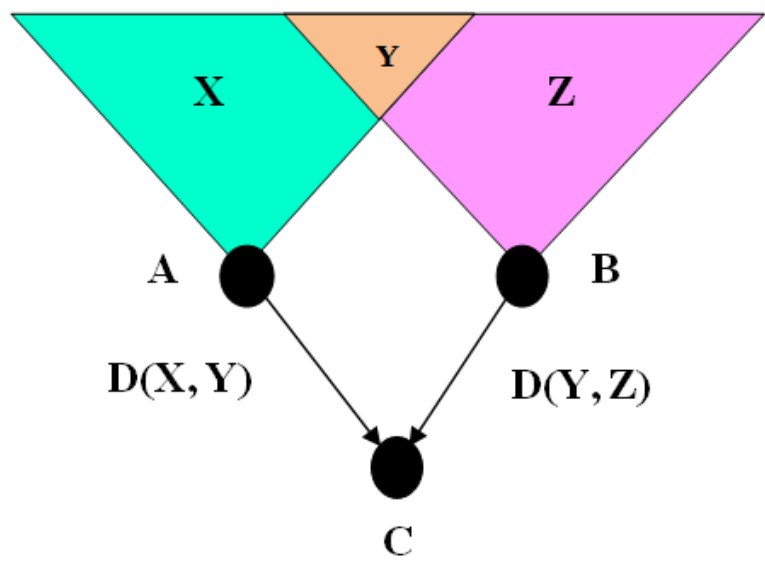

Figure 4. Overlapping Problem: The Node $C$ Receives the same Data $Y$ by both $A$ and $B$ [25]

\section{- $\quad$ Resource Blindness}

The flooding algorithm completely ignores the resource constraints of individual nodes. The nodes are not able to modify their operations based on the amount of energy they are having. So the nodes with resource scarcity get destroyed easily.

\section{SPIN (Sensor Protocol for Information via Negotiation)}

SPIN is a data-centric routing protocol [27]. Here the sensor nodes negotiate with each other before transmitting the actual data using meta-data. Meta-data is used by sensor nodes to give a complete description of the data that they collect and their size should be less than the actual data in terms of bytes. It is basically a three-way handshake protocol which uses three types of messages ADV, REQ and DATA [9-29]. The SPIN family is designed to address the deficiencies of flooding. Problems like implosion and overlapping are solved by negotiation and resource blindness is overcome by resource adaptation, thus achieving energy efficiency. The advantage of this protocol is that each node needs to know only its single-hop neighbours. It saves more energy than flooding [14], and metadata negotiation reduces the redundant data. However, SPIN's data advertisement mechanism does not always guarantee data delivery. The SPIN family of protocols includes many protocols [29]. These include SPIN-PP, SPIN-EC, SPIN-BC and SPINRL.

\section{- $\quad$ SPIN-PP}

This protocol is designed for a point to point communication. Here two nodes can communicate with each other without interfering with other nodes. It uses negotiation to 
solve the problems of flooding and follows 3-way handshake protocol [27]. When a SPIN node receives a new data, it broadcasts an ADV message to its neighbour nodes, which contains the meta-data. If the neighbour does not have the data and needs the data, it sends a REQ message to request data stating which advertised data it wants and does not want. Hence the sender node can differentiate between lost ADV messages and ADV for requested data. DATA is the actual message which is sent to the requesting node. This neighbour sensor node then repeats this process with its neighbours. This way the entire sensor area will receive a copy of the data.

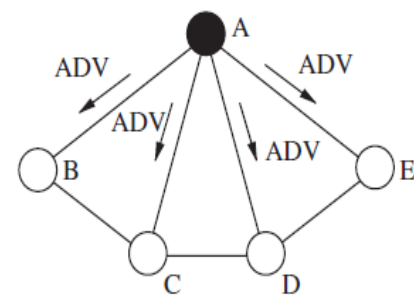

(a)

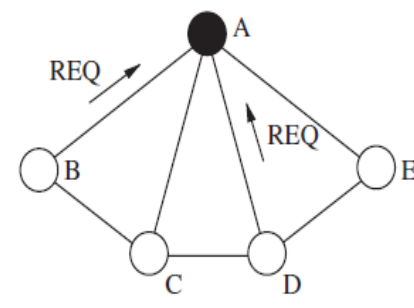

(b)

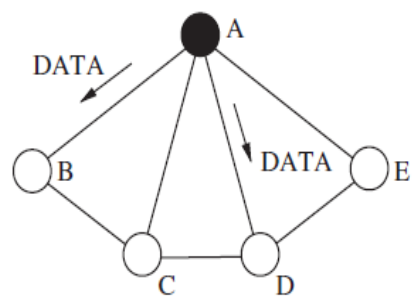

(c)

\section{Figure 5. The SPIN-PP Protocol: (a) Advertisement Phase, (b) Request Phase, and (c) Data Transmission. [5]}

\section{- $\quad$ SPIN- EC}

Here the sensor nodes communicate using the same 3-way handshake protocol as in case of SPIN-PP but there is energy conservation parameter added to it [26]. After getting the ADV message the nodes will check whether its energy is above or below the threshold energy.

If it is above the energy threshold it will perform the operations similar to SPIN-PP. If it is below the threshold energy it will not send the REQ message and hence will not participate in the communication.

\section{- $\quad$ SPIN-BC}

This protocol is designed for broadcast channels nodes where the networks communicate using a single, shared channel [7]. Here when a node sends out a message, it is received by every node irrespective of the message destination. If a node wishes to send a message and senses that the channel is currently in use, it must wait for the channel to become idle before attempting to send the message. Therefore it wastes both time and energy. However, the advantage of such networks is that when a single node sends a message out to a broadcast address, this message can reach all of the node's neighbours using only one transmission. One-to-many communication is therefore $1 / n$ times cheaper in a broadcast network than in a point-to-point network, where $n$ is the number of neighbours for each node.

\section{- SPIN-RL}

It is similar to SPIN-BC protocol but more reliable. This is achieved by adding few changes to SPIN-BC protocol. Here each node keeps track of all the advertisements and the nodes sending them. If it does not receive any requested data within a certain period of time, it sends out the request again. The nodes also have limited frequency with which 
they resend the data messages. After sending out a data message, a node will wait for a certain period of time before it responds to other requests for the same data message. 
Table 1. Brief Idea About SPIN and its Various Types [2][10]

\begin{tabular}{|l|l|}
\hline $\begin{array}{l}\text { SPIN AND } \\
\text { ITS TYPES }\end{array}$ & CHARACTERISTICS \\
\hline SPIN & Uses Data Negotiation and Resource Adaptive Algorithm \\
\hline SPIN-PP & Point to Point Transmission Media \\
\hline SPIN-EC & Adds Energy Conservation Heuristic to SPIN-PP \\
\hline SPIN-BC & Single Broadcast Transmission Media \\
\hline SPIN-RL & Reliable version of SPIN-BC \\
\hline
\end{tabular}

\section{Related Work}

1. Devender Sharma, Harkesh Sehrawat, Jyoti [2012] proposed "Energy Efficient MSPIN Protocol" [4].

In this paper emphasis was given on one of the most challenging aspect of WSN i.e. Energy Efficiency. Different routing protocols have been proposed to save energy during data transmission in the past. In this paper an algorithm M-SPIN has been proposed for increasing the energy efficiency of routing protocol. M-SPIN was already implemented but it suffered from energy problem since several nodes are traversed multiple times resulting in elimination of those nodes from the network. To solve this issue, energy level was used as a parameter. It was found that the energy efficiency was increased but it had complex computations like calculating energy at each node and every time.

2. Geetu, Sonia Juneja [2012] proposed "Performance Analysis of SPIN and LEACH Routing Protocol in WSN" [6].

The aim was to compare the performance of two routing protocols - SPIN and LEACH using data centric approach. And hence the best routing protocol for WSN was proposed. The simulation was based on energy performance using Network Simulator 2.34. The simulation was run for 2 minutes using 50 nodes for both protocols. It was observed that SPIN fares much better than LEACH in terms of energy consumption. Also the end to end delay and dead nodes is more in case of LEACH but the packet delivery ratio is more in case of LEACH as there is no time boundation.

3. K. Karthikeyan, M.Kavitha [2013] proposed "Comparative Analysis of Data Centric Routing Protocols for Wireless Sensor Networks" [13].

This paper compares the three data centric routing protocols SPIN, SPIN-1, M-SPIN for energy efficiency. Based on this analysis M-SPIN performs better than other two. MSPIN is a better approach for the application need quick and reliable response. But one major problem in M-SPIN is that few sensor nodes may be used several times and those nodes may dissipate energy and may be destroyed earlier than other nodes in the network. Cluster methodology and dynamic cluster head election can be used in future to overcome the problem of using only few nodes for the forwarding of the data. The network simulator version 2 was used for performances analysis.

4. Parul Tyagi, Surabhi Jain [2012] proposed "Comparative Study of Routing Protocols in Wireless Sensor Network" [19].

In this paper, recent routing protocols for wireless sensor network has been analyzed and classified in three types of approaches according to network architecture in WSN. The three main categories on the basis of network structure are Flat, Hierarchical and location based routing protocols. The tradeoff between energy and communication overhead savings in every routing protocol was also studied. The advantages and performance issues of each routing technique has been discussed. 


\section{Performance Analysis}

This section compares the two routing protocols - Flooding and SPIN. Flooding is a routing protocol in which each node forwards data to all its neighbour nodes except the node which has sent the data. This process is repeated until all the nodes have received the packets. The advantage of flooding is its simplicity along with guaranteed data delivery. However it encounters many problems like implosion, overlapping and resource blindness which causes transmission of redundant data and wastage of energy and bandwidth [28].

SPIN is a negotiation based protocol suitable for wireless sensor networks. Every node uses meta-data to give description about the data it is going to send. This prevents redundant data transmission in the network. Negotiation is done by sensor nodes before sending data. It performs three way handshaking to send data to its neighbour nodes. Only required data is sent to the neighbour nodes thus preventing duplicate packets and saving energy [6]. The negotiations are done by exchanging data advertisement message (ADV) and a request for data message (REQ) between the sender and the receiver. After the negotiation, the sender transmits the data to the receiver (DATA) only after receiving the REQ message. Hence it is energy efficient though data delivery is not guaranteed here. To solve this issue some changes can be done in the existing protocol by enhancing some of its existing features.

In the table given below, comparison has been made between the two protocols based on various parameters. This analysis is based on various researches done in this field.

Table 2. Comparison Between Flooding and SPIN [10][21][22]

\begin{tabular}{|l|l|l|}
\hline HEURISTICS & FLOODING & \multicolumn{1}{|c|}{ SPIN } \\
\hline Network Topology & Information not required & Required \\
\hline Data Delivery & Guaranteed & Not Guaranteed \\
\hline Redundant Data & Present & Not Present \\
\hline Energy Consumption & High & Low \\
\hline Latency & High & Low \\
\hline More number of nodes & Preferred & Not Preferred \\
\hline Query Based & No & Yes \\
\hline Throughput & Low & High \\
\hline Reliability & Low & High \\
\hline Lifetime of nodes & Low & High \\
\hline Energy Efficiency & Low & High \\
\hline Energy & Yes \\
Awareness & No & \\
\hline
\end{tabular}

\section{Conclusion}

In this paper Flooding has been introduced as a simple routing protocol which is widely used for data transmission. However it suffers from many disadvantages like implosion, overlapping and resource blindness. In order to overcome the above disadvantages, SPIN protocol is used [11]. It is data centric and uses meta-data negotiation before transmitting the actual data. Hence it prevents redundant data transmission to a great extent. It has been analysed that flooding can be used when there are large number of nodes. But in most cases it consumes large amount of energy, resulting in energy wastage by creating huge volume of redundant data. So instead of using flooding, SPIN can be used which will increase the throughput and reliability, while reducing the latency. To reduce the energy consumption further, different types of SPIN 
protocols can be used. It is seen that SPIN-EC and SPIN-RL saves a large amount of energy [10].

In the future work simulated results will be given to show the advantages of SPIN over flooding in various scenarios. Though the SPIN protocol is energy efficient but it does not guarantee data delivery. There may be cases where a node between the source and the destination is not interested in the ADV packet so the actual data may not reach the destination. Furthermore there may be situations when few sensor nodes may be used several times and those nodes may dissipate energy and may be destroyed earlier then other nodes. To avoid these problems one of the solutions can be an additional destination field added to the header and each node in the network will maintain a list of its neighbours. So each time a node gets the ADV packet, it will check whether the destination lies in the list of its neighbours. Moreover, after receiving the ADV packet, the nodes will respond with a REQ message along with a flag which is set to 0 if it does not have the data and 1 if it already has the data, along with its energy value. Then, depending on the largest energy value, the data will be sent to the corresponding node. There by preventing the nodes with lower energy value from getting exhausted easily. A lot of study can be done in this field to overcome the issues of SPIN and make it a preferred protocol for routing in WSN.

\section{Acknowledgments}

We take this opportunity to express our gratitude to all the people who helped and supported us to complete this thesis. We express our sincere thanks to Dr. Kalpana Sharma, Head of Computer Science \& Engineering Department and professor in Computer Science \& Engineering, for providing us invaluable support and able guidance throughout the thesis. She has been a source of inspiration for us that helped us and motivated us throughout.

\section{References}

[1] H. Azni, M. M. Saudi, A. Azman and A. S Johari, "Performance Analysis of Routing Protocol for WSN Using Data Centric Approach", World Academy of Science, Engineering and Technology, vol. 53, (2009).

[2] A. Perrig, R. Szewzyk, J. D. Tygar, V. Wen, and D. E. Culler, "SPINS: security protocols for sensor networks", Wireless Networks, vol. 8, (2000), pp. 521-534.

[3] C.-Y.Chong and P. Srikanta, "Sensor Networks: Evolution, Opportunities, and Challenges", IEEE Tranactions on sensor networks, vol. 28, (2005), pp.117-123.

[4] D. Sharma, H. Sehrawat and Jyoti, "Energy Efficient M-SPIN Protocol”, International Journal of Scientific \& Engineering Research, vol. 3, is.10, (2012) October.

[5] S. C. Ergen and P. Varaiya. "Energy Efficient Routing with Delay Guarantee for SensorNetworks", ACM Wireless Networks, vol. 13, (2007), pp. $679-690$.

[6] Geetu and S. Junega, "Performance Analysis of SPIN and LEACH Routing Protocol in WSN", International Journal Of Computational Engineering Research (ijceronline.com), vol. 2, is. 5.

[7] H. Hassanein and J. Luo, "Reliable Energy Aware Routing in Wireless Sensor Networks", In: Second IEEE Workshop on Dependability and Security in Sensor Networks and Systems, (2006), pp.54-64.

[8] I. F. Akyildiz, W. Su, Y. S. Subramaniam and E. Cayirci, "Wireless sensor networks: a survey", Comp. Netw., (2002), pp. 393-422.

[9] J. N. Al-Karaki and A.E. Kamal, "Routing Techniques in Wireless Sensor Networks", A Survey. In: IEEE Wireless Communications, vol. 11, no. 6, (2004), pp. 6-28.

[10] J. Kulik, W.R. Heinzelman and H. Balakrishnan, "Negotiation-Based Protocols for Disseminating Information in Wireless Sensor Networks", In: Wireless Networks, vol 8, (2002), pp. 169-185.

[11] K. C. Rahman, "A Survey on Sensor Network", JCIT, ISSN 2078-5828 (PRINT), ISSN 2218-5224 (ONLINE), vol. 01, is. 01, MANUSCRIPT CODE: 100715, (2010).

[12] K. Akkaya and M. Younis, "A Survey On Routing Protocols For Wireless Sensor Networks", Elsevier Ad Hoc Network Journal, vol. 3, (2005), pp. 325-349.

[13] K. Karthikeyan and M. Kavitha, "Comparative Analysis of Data Centric Routing Protocols for Wireless Sensor Networks", International Journal of Scientific and Research Publications, vol. 3, no. 1, (2013) January. 
[14] K. Padmanabhan, "A Study on Energy Efficient Routing Protocols in Wireless Sensor Networks", European Journal of Scientific Research ISSN 1450-216X, Euro Journals Publishing, Inc, vol.60, no.4, (2011), pp. 499-511.

[15] R. R. Mudholkar, S. R. Sawant and V. C. Patil, "Classification and comparsion of routing protocols in wireless sensor networks", Ubicc journal, vol. 4, (2012), pp.704-711.

[16] B. Munish, D. K. Kotary and S. Soni, "Comparative analysis of energy efficient routing protocol for wireless sensor network", International journal of computer application on wireless communication and mobile networks, no. 14, (2012), pp.65-69.

[17] K. Padmanabhan and P. Kamalakkannan, "A Study on Energy Efficient Routing Protocols in Wireless Sensor Networks", European Journal of Scientific Research, vol. 60, no. 4, (2011), pp. 499-511.

[18] P. P. Bhattacharya, J. Saraswat and N. Rathi, "A Review on Routing Protocols for Application in Wireless Sensor Networks", International Journal of Distributed and Parallel Systems, vol. 3, no. 5, (2012).

[19] Parul Tyagi and Surabhi Jain, "Comparative Study of Routing Protocols in Wireless Sensor Network", International Journal of Advanced Research in Computer Science and Software Engineering, Volume 2, Issue 9, September 2012.

[20] V. Rajashree, V. C. Biradar, S. R. Sawant and R. R. Mudholkar, "Classification and comparison of Routing Protocols in Wireless Sensor Networks", Ubiquitous Computing Security Systems Journal, vol. 4, (2012), pp. 704-711.

[21] Y. B. Shi, X. B. Ye and P. L. Liu, "Research status on wireless sensor networks", Foreign Electronic Measurement Technology, vol. 24, no. 1, (2005), pp. 19-23.

[22] V. Srinivasan, G. Saritha and T. Saravanan, "A Analysis of Flat Routing Protocols in Sensor N/W", Middle-East Journal of Scientific Research, ISSN 1990-9233,@ IDOSI Publications, (2014).

[23] Y. Zhang and M. Fromherz, "A Robust and Efficient Flooding-Based Routing for Wireless Sensor Networks," Journal of Interconnection Networks, vol. 7, no.4, (2006), pp. 549-568.

[24] Z. Rehena, S. Roy and N. Mukherjee ,"A Modified SPIN for Wireless Sensor Networks", 978-1-4244 8953-4/11, IEEE, (2011).

[25] D. Waltenegus, "Fundamentals of wireless sensor networks : theory and practice / Waltenegus Dargie", Christian Poellabauer, ISBN 978-0-470-99765-9, (2010), pp. 168-172.

[26] L. $\mathrm{Li}$ and F. Wu, "Research on SPIN of wireless sensor network", Computer and Modernization, vol. 3, (2007), pp. 93-96.

[27] S. Basagni, "Distributive and Mobility-Adaptive Clustering for Multimedia Support in Multi-hop Wireless Networks", Proceedings of Vehicular Technology Conference, VTC, vol. 2, (1999), pp. 889893.

[28] C. Schurgers and M. B. Srivastava, "Energy Efficient Routing in Wireless Sensor Networks", MILCOM Proceedings on Communications for Network-Centric Operations: Creating the Information Force, McLean, VA, (2001).

[29] W. R. Heinzelman, J. Kulik and H. Balakrishnan, "Adaptive Protocols for Information Dissemination in Wireless Sensor Networks," Proceedings of the 5th Annual ACM/IEEE Conference on Mobile Computing and Networking, Seattle, Washington, United States, (1999) August 15-19, pp. 174- 185.

\section{Authors}

Dr. Kalpana Sharma- is an Associate Professor of the Department of Computer Science \& Engineering at Sikkim Manipal. Institute of Technology, Mazitar, Sikkim, India since August, 1998. She did her BE from National Institute of Technology, Silchar, India and M.Tech from IIT Kharagpur, India. She completed her PhD on 2011 and her topic of research was security in wireless sensor networks. Her areas of research interest are Wireless Sensor Networks, Steganography, Network \& Information Security, Real Time Systems and Software Engineering. She has published a number of technical papers in various national and international journals in addition to presentation/ publication in several international/ national conferences. She can be reached at kalpanaiitkgp@yahoo.com.

Neha Mittal- is a final year B. Tech student of Computer Science and Engineering Department at Sikkim Manipal Institute and Technology. Her areas of interest are Wireless Sensor Networks and Ad hoc Networks. She can be reached at nehamittal135@gmail.com. 
Priyanka Rathi- is a final year B. Tech student of Computer Science and Engineering Department at Sikkim Manipal Institute and Technology. Her areas of interest are Wireless Sensor Networks and Ad hoc Networks. She can be reached at priyanka.rathi7792@gmail.com. 